\title{
Validation of Dimensionless Parameters for Distinguishing between Homogeneous and Bubbling Fluidizations
}

\author{
Kenya Kuwagi1, Atsuto Kogane, Yui Sasaki', Hiroyuki Hirano² \\ ${ }^{1}$ Department of Mechanical Systems Engineering, Okayama University of Science, Okayama, Japan \\ ${ }^{2}$ Department of Applied Chemistry and Biotechnology, Okayama University of Science, Okayama, Japan \\ Email: kuwagi@mech.ous.ac.jp
}

How to cite this paper: Kuwagi, K., Kogane, A., Sasaki, Y. and Hirano, H. (2021) Validation of Dimensionless Parameters for Distinguishing between Homogeneous and Bubbling Fluidizations. Open Journal of Fluid Dynamics, 11, 81-97.

https://doi.org/10.4236/ojfd.2021.112006

Received: March 26, 2021

Accepted: June 8, 2021

Published: June 11, 2021

Copyright $\odot 2021$ by author(s) and Scientific Research Publishing Inc. This work is licensed under the Creative Commons Attribution International License (CC BY 4.0).

http://creativecommons.org/licenses/by/4.0/

\begin{abstract}
The difference between homogeneous and bubbling fluidization behaviors has been studied for the past 70 years, where several researchers have reported on the influence of interparticle forces in fluidization. Although interparticle forces such as van der Waals forces are evident in a real system, these forces are not the determinant in homogeneous fluidization, which can be simulated without any interparticle forces. In our previous study, the difference in fundamental mechanisms of the two fluidization states was analytically determined with a dimensionless gravity term, comprising the Reynolds number, Archimedes number, and density ratio. Nevertheless, some researchers insist that interparticle forces are dominant and a hydrodynamic force is not dominant. In this study, a dimensional analysis was applied to obtain a dominant parameter for distinguishing two fluidizations. Furthermore, some parameters were examined by comparing the experimental data in previous studies. The results indicated that hydrodynamic force is the dominant factor and the dimensionless gravity term is the dominant parameter in differentiating the two fluidized states.
\end{abstract}

\section{Keywords}

Bubbling Fluidization, Homogeneous Fluidization, Aggregative, Particulate, Dimensional Analysis

\section{Introduction}

\subsection{Research Background}

Fluidization can be observed in several natural phenomena such as avalanches, 
sandstorms, pyroclastic flows, and ground liquefaction. This characteristic is utilized in industrial processes as fluidized beds, where a balance between the drag and gravitational forces acting on the particle bed fluidizes the particles when the fluid velocity from the bottom wall exceeds a certain limit. The velocity at this instant is termed the minimum fluidization velocity, $u_{\mathrm{mf}}$.

There are two kinds of fluidization: homogenous or particulate fluidization, where the particle bed expands homogeneously and bubbling or aggregative fluidization, where voids (bubbles) occur in the particle bed as the injected fluid velocity increases. Geldart [1] classified particles into Groups C, A, B, and D, in ascending order of particle size based on fluidization behavior. The powder bed of Group A homogeneously fluidized above the $u_{m}$ and bubbles were observed at a certain fluid velocity, which is called the minimum bubbling fluidization velocity, $u_{m b}$. However, homogeneous fluidization did not occur in the powder beds of Groups B and D. Moreover, bubbling fluidization is generally not observed in liquid systems such as glass bead-water systems.

\subsection{Literature Review}

As summarized in Table 1, two approaches have been widely used to address the abovementioned issue. The first approach derives a discriminant equation to determine the criteria for the transition between homogeneous and bubbling fluidizations. The first discriminant equation was derived by Wilhelm and Kwauk [2], and numerous criteria have been proposed in the last 60 years [3] [4] [5] [6] [7]. Notably, most of these studies used Froude or Reynolds numbers based on the minimum fluidization velocity to estimate the fluidization state. Although the state of homogeneous or bubbling fluidization can be estimated under each condition, the estimation of the minimum bubbling fluidization velocity with these methods is difficult.

The second approach derives an equation to estimate the minimum bubbling fluidization velocity $u_{m b}$. Primarily, Geldart [1] derived the following simple equation:

$$
u_{m b}=100 d_{p} .
$$

Subsequently, Abrahamsen and Geldart [8] obtained the following equation based on an extensive dataset:

$$
u_{m b}=\frac{2.07 d_{p} \rho_{f}^{0.06}}{\mu_{f}^{0.347}} \exp \left(0.716 F_{45}\right),
$$

where $F_{45}$ is the mass fraction of particles with diameters less than $45 \mu \mathrm{m}$. The equation derived by Abrahamsen and Geldart [8] is frequently used to study gas-fluidized beds. Although they used dimensional analysis, the derived equation was not expressed using dimensionless parameters. Kuwagi et al. [9] conducted a dimensional analysis using the governing equations of discrete element method (DEM) and computational fluid dynamics (CFD) coupling model [10] to describe the phenomenon of fluidization. They plotted the fluidization state 
Table 1. Research on the difference between homogeneous and bubbling fluidization.

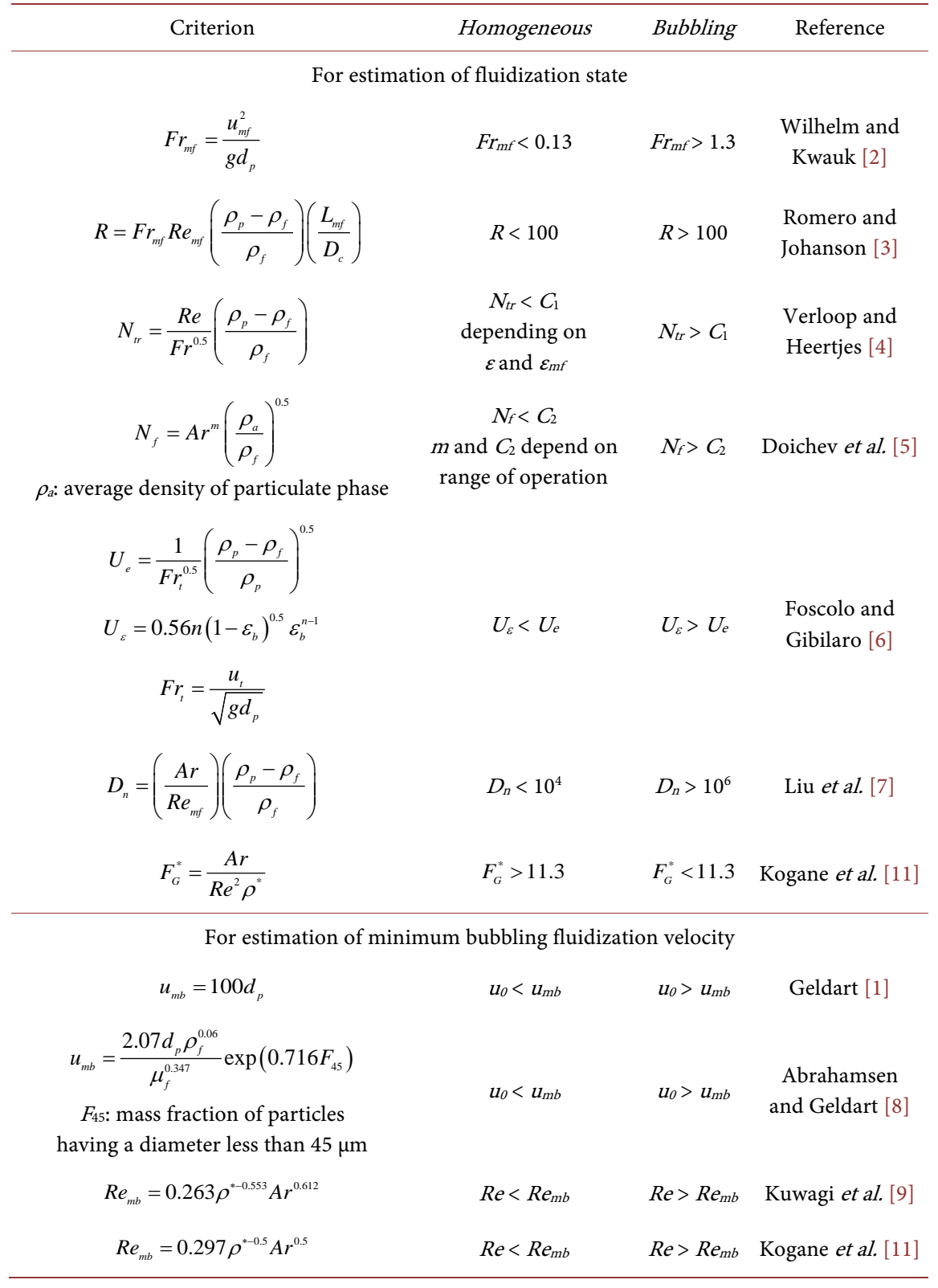

on a three-dimensional (3-D) graph consisting of three dimensionless numbers- $R e, A r$, and $\rho^{*}$. Thus, the following equation was derived from the boundary plane between homogeneous and bubbling fluidization states:

$$
R e_{m b}=0.263 \rho^{*-0.553} A r^{0.612} \text {. }
$$

The derivation method is similar to that of Abrahamsen and Geldart [8] because the fitting equation was obtained from an extensive simulation and experimental data. In contrast, Kogane et al. [11] revealed that the dimensionless gravity term, $F_{G}^{*}=\operatorname{Ar} /\left(\operatorname{Re}^{2} \rho^{*}\right)$, distinguishing the two states of fluidization can be rewritten as

$$
R e_{m b}=0.297 \rho^{*-1 / 2} A r^{1 / 2} .
$$




\subsection{Problems}

Certain explanations have been proposed to adequately clarify the basic mechanisms that differentiate homogeneous and bubbling fluidization. Researchers [12] [13] have argued on the influence of interparticle forces and the insignificant effect of hydrodynamics. Although we can agree with this opinion when treating cohesive powders, Di Renzo and Di Maio [14] and Thornton et al. [15] have successfully simulated homogeneous fluidization without any cohesive forces. Thus, interparticle forces do not seem to be a determining parameter for fluidization. Nevertheless, we again emphasize on considering interparticle forces, such as van der Waals forces, during simulation of cohesive powder, i.e., Group C of Geldart's classification. In other words, interparticle forces are an additional distinguishing factor between the two fluidization states. In addition, homogeneous fluidization can occur in a liquid system with large particles, which is difficult to explain from the standpoint of cohesive forces.

In this study, we used larger particles, i.e., Groups A, B, and D to determine the differences between the two fluidization states. The interparticle force includes a repulsion force resulting from the collision of particles, and the repulsion force can be calculated in a numerical simulation using softer springs to reduce computational time [10]. Nonetheless, the simulated results accurately described the real phenomenon for noncohesive fluidized particles [10]. Ye et al. [13] used a Hookean spring model with $k_{n}=7$ or $3.5 \mathrm{~N} / \mathrm{m}$ for calculating the repulsion force, whereas $k_{n}=800 \mathrm{~N} / \mathrm{m}$ in the current study. In addition, Thornton et al. [15] used $E=100 \mathrm{MPa}$ in a Hertzian spring model. Notably, these studies successfully simulated the difference between homogeneous and bubbling fluidizations. Thus, the repulsion force between particles would not dominantly influence the differences between the two fluidizations.

Furthermore, as mentioned before, dimensional analysis has not been effectively utilized in previous studies for derivation of the discriminant equation to determine the criterion for transition between homogeneous and bubbling fluidizations. When the Froude number or Reynolds number is defined using the minimum fluidization velocity as a reference velocity, the dimensionless number is treated as a physical property, such as the Prandtl number. On the other hand, the velocity of the injected fluid, i.e., the fluidizing velocity, can be used as a reference velocity for the dimensionless numbers. Valverde et al. [12] and Ye et al. [13] concluded that the criterion with the Froude number [2] fails to distinguish between the two fluidized states. In this study, we attempt to alter this perspective by defining the Froude number with the fluidizing velocity instead of the minimum fluidization velocity.

\subsection{Purpose}

The analogy between the dimensional and dimensionless simulations has already been presented [9]. In the study, a 3-D graph incorporating three dimensionless numbers- $R e, A r$, and $\rho^{*}$-showed a clear boundary between the two states of 
fluidization. The dimensionless gravity term included these dimensionless numbers and clearly distinguished the two fluidization states in our previous study [11]. Furthermore, the results were in good agreement with prior experimental data. The definition of the dimensionless gravity term was examined in this study to form a basis of understanding and confirm the applicability of dimensional analysis on fluidization.

\section{Analyses}

\subsection{Dimensional Analysis}

The governing equations for the DEM-CFD coupling model [10] were assigned to equations describing the fluidization phenomenon.

Fluid phase:

$$
\begin{gathered}
\frac{\partial \varepsilon}{\partial t}+\frac{\partial}{\partial x_{i}}\left(\varepsilon u_{i}\right)=0 \\
\rho_{f} \frac{\partial}{\partial t}\left(\varepsilon u_{i}\right)+\rho_{f} \frac{\partial}{\partial x_{i}}\left(\varepsilon u_{i} u_{j}\right)=-\varepsilon \frac{\partial p}{\partial x_{i}}-\varepsilon f_{p f}-\rho_{f} \varepsilon g .
\end{gathered}
$$

Particle phase:

$$
\begin{gathered}
m_{p} \frac{\mathrm{d} \boldsymbol{v}}{\mathrm{d} t}=-V_{p} \nabla p+\left(\boldsymbol{F}_{p p}+\boldsymbol{F}_{p f}\right)-m_{p} \boldsymbol{g}, \\
I \frac{\mathrm{d} \omega}{\mathrm{d} t}=\left|F_{t}\right| r_{p} .
\end{gathered}
$$

There are two definitions of pressure: pressure perturbation, $p^{\prime}$, and the summation of the pressure in the hydrostatic equilibrium and the pressure perturbation, $p$. Equations (6) and (7) are expressed with terms in relation to $p$.

However, Equations (6) and (7) can be expressed with terms in relation to $p^{\prime}$ as

$$
\begin{aligned}
& \rho_{f} \frac{\partial}{\partial t}\left(\varepsilon u_{i}\right)+\rho_{f} \frac{\partial}{\partial x_{i}}\left(\varepsilon u_{i} u_{j}\right)=-\varepsilon \frac{\partial p^{\prime}}{\partial x_{i}}-\varepsilon f_{p f}, \\
& m_{p} \frac{\mathrm{d} \boldsymbol{v}}{\mathrm{d} t}=-V_{p} \nabla p^{\prime}+\left(\boldsymbol{F}_{p p}+\boldsymbol{F}_{p f}\right)-\left(m_{p}-m_{f}\right) \boldsymbol{g} .
\end{aligned}
$$

Thereafter, the following equations were derived by nondimensionalizing Equations (5)-(8).

Fluid phase:

$$
\begin{gathered}
\frac{\partial \varepsilon}{\partial T}+\frac{\partial}{\partial X_{i}}\left(\varepsilon U_{i}\right)=0 \\
\frac{\partial}{\partial T}\left(\varepsilon U_{i}\right)+\frac{\partial}{\partial X_{i}}\left(\varepsilon U_{i} U_{j}\right)=-\varepsilon \frac{\partial P}{\partial X_{i}}-\varepsilon f_{p f}^{*}-\frac{\varepsilon}{F r^{2}} .
\end{gathered}
$$

Particle phase:

$$
\frac{\mathrm{D} \boldsymbol{V}}{\mathrm{d} T}=-\frac{1}{\rho^{*}} \nabla P+\left(\boldsymbol{F}_{p p}^{*}+\boldsymbol{F}_{p f}^{*}\right)-\frac{G a}{R e^{2}} \cdot \frac{\boldsymbol{g}}{|\boldsymbol{g}|},
$$




$$
\frac{\mathrm{d} \omega^{*}}{\mathrm{~d} T}=\left|F_{t}^{*}\right| R
$$

Furthermore, Equations (9) and (10) can be rewritten as

$$
\begin{aligned}
& \frac{\partial}{\partial T}\left(\varepsilon U_{i}\right)+\frac{\partial}{\partial X_{i}}\left(\varepsilon U_{i} U_{j}\right)=-\varepsilon \frac{\partial P^{\prime}}{\partial X_{i}}-\varepsilon f_{p f}^{*}, \\
& \frac{\mathrm{D} \boldsymbol{V}}{\mathrm{d} T}=-\frac{1}{\rho^{*}} \nabla P^{\prime}+\left(\boldsymbol{F}_{p p}^{*}+\boldsymbol{F}_{p f}^{*}\right)-\frac{1}{\rho^{*}} \cdot \frac{A r}{R e^{2}} \cdot \frac{\boldsymbol{g}}{|\boldsymbol{g}|} .
\end{aligned}
$$

In addition, the following reference qualities were used in the nondimensionalization process:

$$
\begin{gathered}
x_{0}=d_{p}, v_{0}=u_{0}, \quad p_{0}=\rho_{f} u_{0}^{2}, t_{0}=\frac{d_{p}}{u_{0}}, \\
f_{0}=\frac{\rho_{f} u_{0}^{2}}{d_{p}}, F_{0}=\frac{\pi}{6} \rho_{p} d_{p}^{2} u_{0}^{2}, \omega_{0}=\frac{10 u_{0}}{d_{p}}
\end{gathered}
$$

Further details on the related nondimensionalization procedure can be found in our previous study [9]. The Archimedes number can be confused with the Galilei number [16]. However, the distinction between the two dimensionless numbers can be easily understood from the nondimensionalization procedure. The Archimedes number signifies the buoyancy force in the gravity term of the particle motion equation, whereas the Galilei number considers only the gravity force in the gravity term and the buoyancy as a static force, thus reflecting the total pressure in the expression.

\subsection{Numerical Simulation}

The fluidization behavior of homogeneous or bubbling fluidization was determined under each condition by performing a DEM-CFD simulation [11], as it was difficult to conduct numerous experiments under various conditions. The simulations were conducted with an in-house code. The accuracy of the simulation code used in this study has been validated in our previous study [9] [11]. The ratio of bed (container) size to particle size was chosen to be constant. The analysis domain is $200 d_{p}$ in width, $700 d_{p}$ in height, and $d_{p}$ in thickness. This indicates that the present simulations are two-dimensional. Since the simulation mesh size was set to $5 d_{p}$, the grid numbers are $40 \times 140$ for the horizontal direction and vertical direction, respectively. The fluid was uniformly injected from the bottom wall at various velocities $u_{0}$.

\subsection{Analysis Conditions}

The simulation conditions are listed in Table 2 and Table 3.

\section{Results and Discussion}

\subsection{Comparison of Estimation Equations}

Equations (3) and (4) were compared with Equations (1) and (2) to be rewritten with dimensional numbers (physical properties) as follows: 
Table 2. Properties of particles.

\begin{tabular}{cc}
\hline Particle diameter: $d_{p}[\mu \mathrm{m}]$ & $50,60,100,200,500,1000$ \\
Particle density: $\rho_{p}\left[\mathrm{~kg} / \mathrm{m}^{3}\right]$ & 265,1000 (FCC), 2650, 5300 (glass), 11,340 (lead) \\
Particle number & 45,000 \\
\hline
\end{tabular}

Table 3. Properties of fluids.

\begin{tabular}{ccc}
\hline Fluid & $\begin{array}{c}\text { Density: } \\
\rho_{f}\left[\mathrm{~kg} / \mathrm{m}^{3}\right]\end{array}$ & $\begin{array}{c}\text { Viscosity: } \\
\mu_{f}[\mathrm{~Pa} \cdot \mathrm{s}]\end{array}$ \\
\hline Air & 1.20 & $1.82 \times 10^{-5}$ \\
Air $(1 \mathrm{MPa})$ & 12.0 & $1.82 \times 10^{-5}$ \\
Air $(10 \mathrm{MPa})$ & 120 & $1.82 \times 10^{-5}$ \\
Air $\left(500^{\circ} \mathrm{C}\right)$ & $4.56 \times 10^{-1}$ & $3.55 \times 10^{-5}$ \\
Water & $9.98 \times 10^{2}$ & $1.00 \times 10^{-3}$ \\
\hline
\end{tabular}

$$
\begin{gathered}
u_{m b}=\frac{1.06 d_{p}^{0.836} \rho_{f}^{0.165}}{\mu_{f}^{0.224}} \times \frac{\left(\rho_{p}-\rho_{f}\right)^{0.612}}{\rho_{p}^{0.553}} \\
u_{m b}=0.930 d_{p}^{0.5}\left(1-\frac{\rho_{f}}{\rho_{p}}\right)^{0.5} .
\end{gathered}
$$

The indices of $d_{p}, \rho_{f}$ and $\mu_{f}$ are listed in Table 4. Although Equation (2) includes the term describing the ratio of fine powder, the indices of $d_{p}$ are 1 and 0.836 , those of $\rho_{f}$ are 0.06 and 0.165 , and those of $\mu_{f}$ are 0.347 and 0.224 in Equations (2) and (18), respectively. Notably, Equation (2) is based on experimental data and Equation (18) is based on simulated data, but the derivation method is similar for fitting the data. When assuming all the fluid property values as constants, Equation (18) becomes a function of $d_{p}^{0.836}$, which is close to the index of 1.0 in Equation (1). Moreover, the absence of the fluid viscosity $\mu_{f}$ term in Equation (19) indicates that the fluid viscosity is not an essential parameter. Furthermore, the fluid density $\rho_{f}$ was expressed only in the buoyancy term: $(1-$ $\rho_{t}\left(\rho_{p}\right)$. Therefore, the form of Equation (19) is similar to Equation (1).

\subsection{Comparisons of Criteria}

Certain equations from Table 1 were compared using our simulated data. The distribution of the Froude number $F r_{m f}$ based on $u_{m f}[2]$ is presented in Figure 1. The blue and red circles indicate homogeneous and bubbling fluidizations, respectively; the filled circles indicate a fixed bed (nonfluidization). The fluidization states plotted in Figure 1 were obtained through simulations. In addition, the minimum bubbling fluidization velocity representing the boundary between homogeneous and bubbling fluidizations measured in previous studies [16] [17] [18] [19] is plotted in Figure 1. Although Wilhelm and Kwauk [2] set the boundary at $F r_{m f}=1$, bubbling fluidization occurs at $F r_{m f}>0.1$, as shown in Figure 1 . Furthermore, the distinction of the fluidization type becomes difficult under 


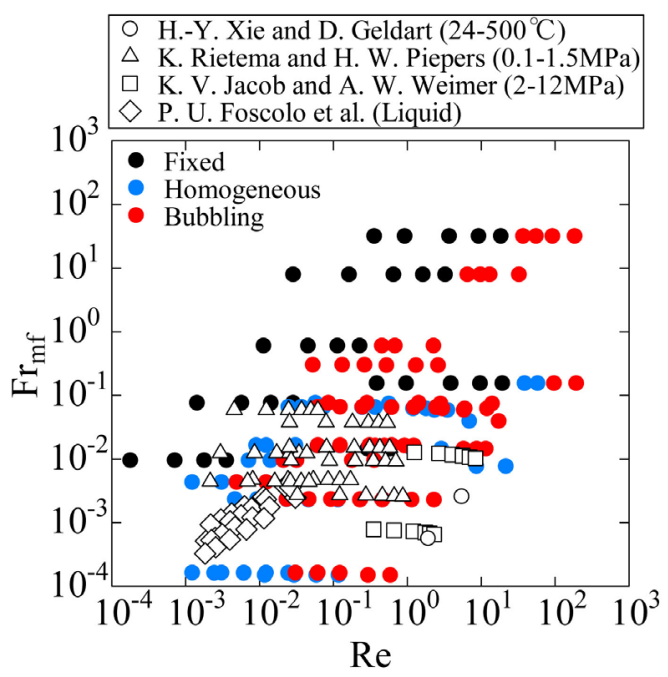

Figure 1. Relationship between Froude numbers based on $u_{m f}[2]$ and fluidization behaviors.

Table 4. Comparison of property indices in $u_{m b}$ equations.

\begin{tabular}{cccc}
\hline Fluid & $d_{p}$ & $\rho_{f}$ & $\mu_{f}$ \\
\hline Geldart (1973) (Equation (1)) & 1 & - & - \\
Abrahamsen and Geldart (1980) (Equation (2)) & 1 & 0.06 & 0.347 \\
Kuwagi et al. (2014) (Equation (3)) & 0.836 & 0.165 & 0.224 \\
Kogane et al. (2019) (Equation (4)) & 0.5 & - & - \\
\hline
\end{tabular}

$F r_{m f}<0.1$. The experimental data obtained from the literature [16] [17] [18] [19] are indicated with vacant symbols in Figure 1 . The boundary between the two fluidization states ranged from $10^{-1}$ to $10^{-3}$ [12]. However, differentiating the two fluidization behaviors from the results was difficult with the Froude number $F r_{m f}$ based on $u_{m}$ Therefore, the conclusion that the Froude number criterion fails to estimate the difference [12] [13] is plausible.

The values of $U_{e}-U_{\varepsilon}$ calculated using the criterion from Foscolo and Gibilaro [6] are depicted in Figure 2, which shows certain outlying formations of bubbling fluidization (red circles) in the region above the dashed line. All the outlying phenomena occurred with particles from Group C of Geldart's classification, indicating that this criterion cannot be applied to particles from Group C. Moreover, Valverde et al. [12] specified that Foscolo and Gibilaro [6] had neglected particle inertia. However, their criterion can distinguish between the two forms of fluidization.

Furthermore, Figure 3 shows the discrimination number $D_{n}$ proposed by Liu et al. [7]. They concluded that homogeneous fluidization occurs at $D_{n}<10^{4}$, transitional region ranges from $10^{4}$ to $10^{6}$, and bubbling fluidization occurs at $D_{n}>10^{6}$. However, the boundary between the bubbling fluidization and transitional states evaluated from our simulation was at $10^{7}$, as shown in Figure 3. Moreover, experimental data existed beyond the order of $10^{7}$. 


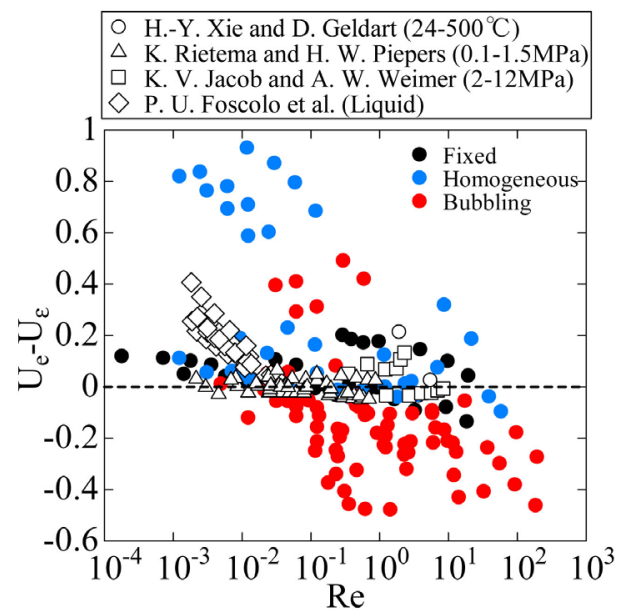

Figure 2. Relationship between the criterion of Foscolo and Gibilaro [6] and fluidization behaviors.

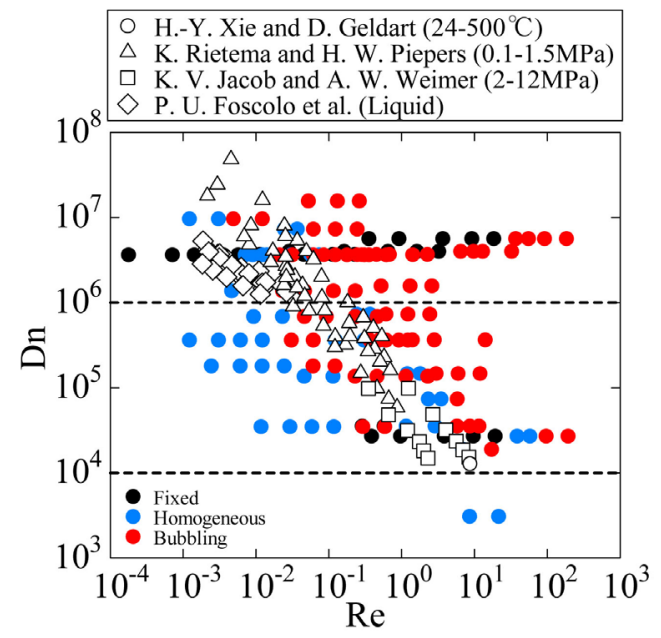

Figure 3. Relationship between discrimination number [7] and fluidization behaviors.

The values of the dimensionless gravity term $F_{G}^{*}$ proposed by the present authors [11] are illustrated in Figure 4. Both $F r_{m f}$ and $D_{n}$ are based on $u_{m 6}$ and therefore cannot be used as the discriminant in varying the fluidizing velocity or superficial velocity $u_{0}$. On the contrary, the dimensionless gravity term is a dimensionless parameter based on $u_{0}$; thus, it can be applied to alter the fluidization pattern by varying $u_{0}$. The homogeneous and bubbling fluidizations were well separated in the obtained results, and the boundary value was consistent with the experimental values of $u_{m b}$ referred from the literature [16] [17] [18] [19]. Upon further examination, the boundary value was found to decrease with an increasing Reynolds number. This tendency is attributed to the assumption of Stokes flow in the derivation of the dimensionless gravity term [11]. In particular, this could be an effect of particle inertia. Thus, we derived a modified dimensionless gravity term [11] as a solution.

In this study, we considered a Froude number based on $u_{0}$ instead of $u_{\mathrm{mf}}$ [2], and the results are plotted in Figure 5. In contrast to the results shown in Figure 1, 


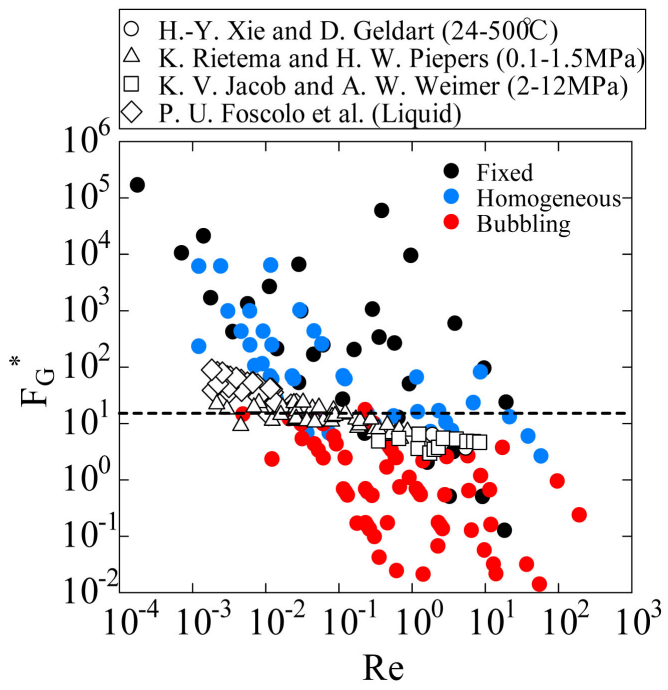

Figure 4. Relationship between dimensionless gravity term [11] and fluidization behaviors.

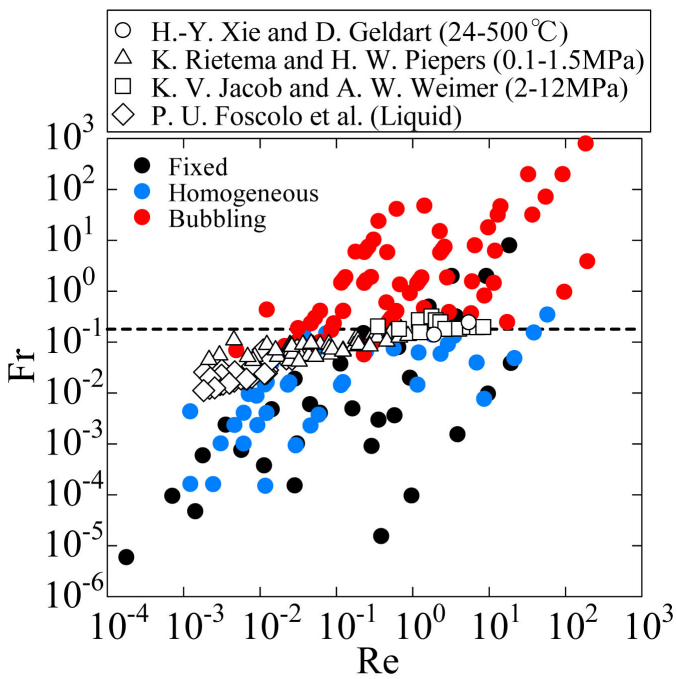

Figure 5. Relationship between Froude numbers based on fluidizing velocity, $u_{0}$, and fluidization behaviors.

the homogeneous and bubbling fluidizations were well separated by a boundary value of 0.1. Moreover, this result is similar to that presented in Figure 4. Furthermore, the relationship between $F r$ and $F_{G}^{*}$ can be expressed using the dimensionless numbers as

$$
\begin{gathered}
F r=\frac{R e^{2}}{G a} \\
F_{G}^{*}=\frac{A r}{R e^{2} \rho^{*}} .
\end{gathered}
$$

These equations correspond to the expressions of gravity in Equations (13) and (16). Therefore, both $F r$ and $F_{G}^{*}$ were derived from the dimensionless governing equations discussed earlier, and the distinction is highlighted from the 
treatment of pressure, i.e., $p$ or $p^{\prime}$, as shown in Equations (7) and (10). Notably, Fr comprises two dimensionless numbers, whereas $F_{G}^{*}$ comprises three dimensionless numbers. The dimensional analysis requires three dimensionless parameters [9], and $F_{G}^{*}$ satisfies this condition but Fr does not. Nevertheless, Fr can distinguish the two fluidization states, as shown in Figure 5, and the reason has to be clarified with further studies.

\subsection{Accuracy of Estimation Equation}

The estimation equation for $u_{m b}$ was quantitatively verified by comparing the experimental values with that referred from the literature [16] [17] [18] [19]. The values calculated using Equations (2), (3), and (4) are presented in Figure 6, Figure 8 and Figure 9, respectively. In addition, the values calculated using the equation of Foscolo and Gibilaro [6] are presented in Figure 7. In each figure, the ordinate indicates the calculated value, whereas the abscissa indicates the experimental value.

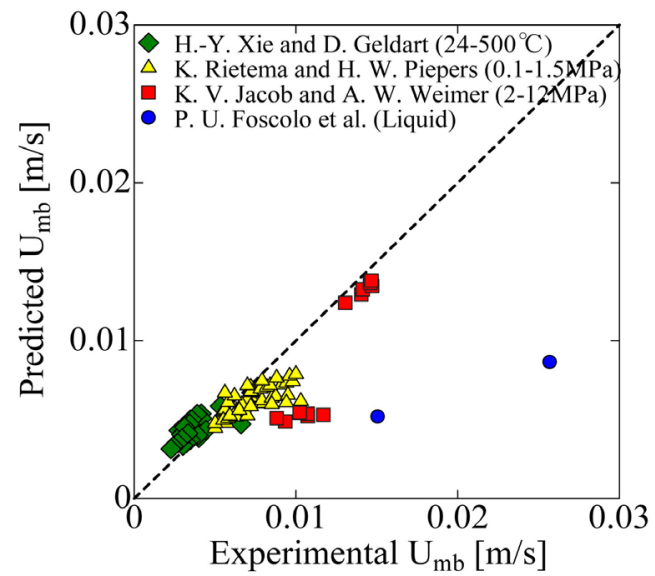

Figure 6. Comparison of $u_{m b}$ values predicted by equation of Abrahamsen and Geldart [8] (Equation (2)) with experimental data.

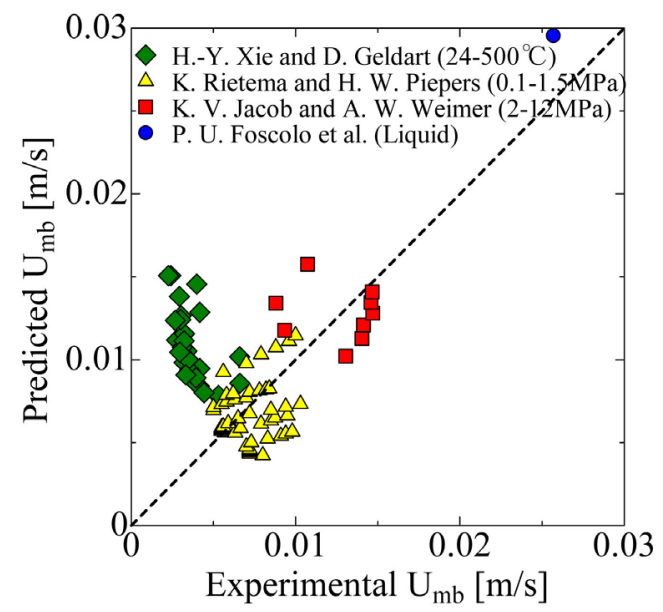

Figure 7. Comparison of $u_{m b}$ values predicted by equation of Foscolo and Gibilaro [6] with experimental data. 
As shown in Figure 6, the values calculated using Equation (2) were in poor agreement with the experimental values for the liquid system (blue circles) because Abrahamsen and Geldart [8] obtained their equation based on data of fluidizing fine powder under gas flow. As shown in Figure 7, the equation of Foscolo and Gibilaro [6] provided a better correlation in the case of the liquid system, but the overall variance was large. Moreover, a considerable agreement can be observed in Figure 8 because Equation (3) was obtained from an extensive dataset comprising 3-D flow regime map [9], including that of a liquid system. Figure 9 displays another disagreement for the liquid system owing to its larger particle diameters and higher fluidizing velocities, i.e., high Reynolds numbers. As the dimensionless gravity term in our study was derived by assuming Stokes flow, we used the modified dimensionless gravity term [11]. However, the dimensionless gravity term should be used to determine the fundamental mechanisms differentiating between homogeneous and bubbling fluidizations. Regardless, the disagreement is unimportant in terms of analyzing the fundamental mechanisms of the two fluidization behaviors.

The deviation from the experimental data is summarized in Table 5. Although Equation (3) can be applied to a wide range of conditions including liquid systems, its application in fine powders without modification is difficult. In Figure 9, the deviation between the predicted and experimental values broadened as $u_{m b}$ increased; a large $u_{m b}$ indicates a high Reynolds number. Moreover, the reason for the deviation is the same as that discussed in Figure 4.

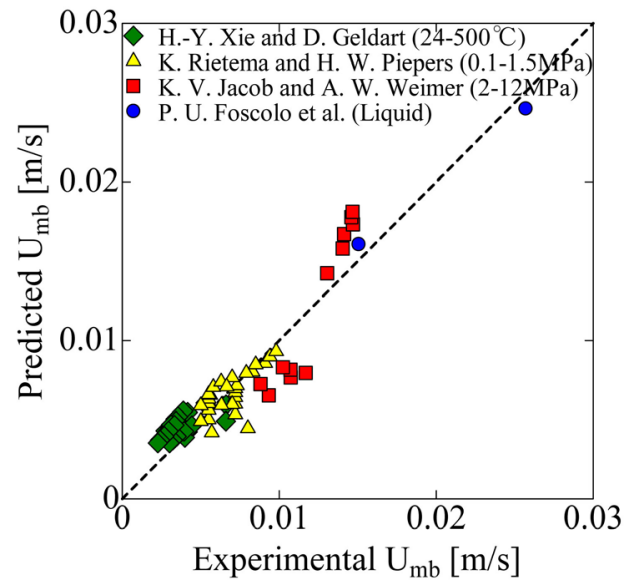

Figure 8. Comparison of $u_{m b}$ values predicted by equation of Kuwagi et al. [9] (Equation (3)) with experimental data.

Table 5. Deviation from experimental data.

\begin{tabular}{cc}
\hline Estimation equation & Deviation from exp. data [\%] \\
\hline Abrahamsen and Geldart [8], Equation (2) & 27 \\
Foscolo and Gibilaro [6] & 39 \\
Kuwagi et al. [9], Equation (3) & 18 \\
Kogane et al. [11], Equation (4) & 33 \\
\hline
\end{tabular}




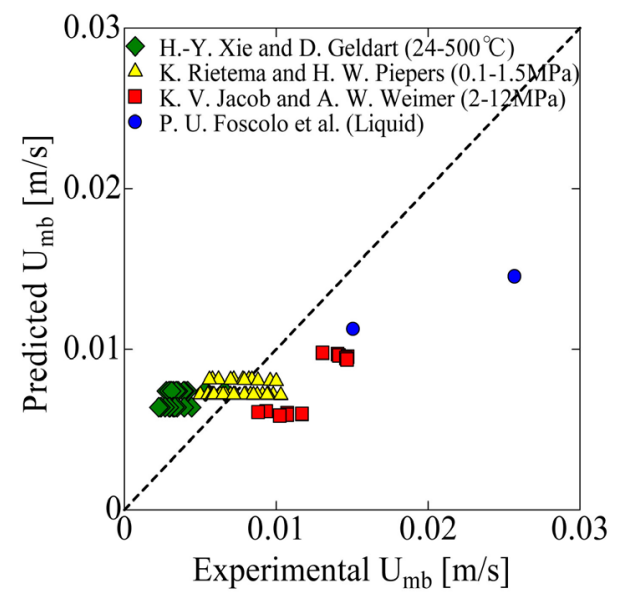

Figure 9. Comparison of $u_{m b}$ values predicted by equation of Kogane et al. [11] (Equation (4)) with experimental data.

\section{Conclusions}

In this study, the distinct fundamental mechanisms of the homogeneous and bubbling fluidization behaviors were determined using a dimensionless gravity term that was derived to distinguish the two fluidization states as per our previous report [11]. The dimensionless gravity term was validated with results of prior research.

The estimation equations for dimensional analysis were derived from a 3-D flow regime map and the dimensionless gravity term was compared with the criteria reported in previous studies. A comparative analysis on the indices of physical properties confirmed that our derived equations were almost equivalent to the equations established in prior research. Thus, the dimensionless gravity term is signified as a dominant parameter for distinguishing between homogeneous and bubbling fluidization. In addition, the dimensionless gravity term was expressed in the dimensionless governing equations. In comparison to interparticle forces, the results of the current research indicated that hydrodynamic force is the most important and dominant factor in differentiating the two fluidized states. However, interparticle forces, such as cohesive and repulsion forces and the effect of particle inertia can be considered as additional factors to demonstrate more accurate simulations. Notably, the distinction between the two fluidization states can be simulated and observed without considering these additional factors as well.

Furthermore, the estimation equations were compared with existing discriminant equations. The minimum bubbling fluidization velocities estimated in the current study were consistent with the experimental data, and the estimation equations accurately distinguished the two fluidization behaviors.

\section{Conflicts of Interest}

The authors declare no conflicts of interest regarding the publication of this paper. 


\section{References}

[1] Geldart, D. (1973) Types of Gas Fluidization. Powder Technology, 7, 285-292. https://doi.org/10.1016/0032-5910(73)80037-3

[2] Wilhelm, R.H. and Kwauk, M. (1948) Fluidization of Solid Particles. Chemical Engineering Progress, 44, 201-218.

[3] Romero, J.B. and Johanson, L.N. (1962) Factors Affecting Fluidized Bed Quality. Chemical Engineering Progress Symposium Series, 58, 28-37.

[4] Verloop, J. and Heertjes, P.M. (1970) Shock Waves as a Criteria for the Transition from Homogeneous to Heterogeneous Fluidization. Chemical Engineering Science, 25, 825-832. https://doi.org/10.1016/0009-2509(70)85117-X

[5] Doichev, K., Todorov, S. and Dimitrov, V. (1975) Transition between Particulate and Aggregative Fluidization at Different State-of-Flow of the Solids. Chemical Engineering Science, 30, 419-424. https://doi.org/10.1016/0009-2509(75)85007-X

[6] Foscolo, P.U. and Gibilaro, L.G. (1984) A Fully Predictive Criterion for the Transition between Particulate and Aggregate Fluidization. Chemical Engineering Science, 39, 1667-1675. https://doi.org/10.1016/0009-2509(84)80100-1

[7] Liu, D., Kwauk, M. and Li, H. (1996) Aggregative and Particulate Fluidization-The Two Extremes of a Continuous Spectrum. Chemical Engineering Science, 51, 4045-4063. https://doi.org/10.1016/0009-2509(96)00247-3

[8] Abrahamsen, A.R. and Geldart, D. (1980) Behavior of Gas-Fluidized Beds of Fine Powders Part I. Homogeneous Expansion. Powder Technology, 2, 35-46. https://doi.org/10.1016/0032-5910(80)85005-4

[9] Kuwagi, K., Kogane, A., Bin Alias, A., Hirano, H. and Takami, T. (2014) Nondimensionalization and Three-Dimensional Flow Regime Map for Fluidization Analyses. Chemical Engineering Science, 119, 199-211. https://doi.org/10.1016/j.ces.2014.08.006

[10] Tsuji, Y., Kawaguchi, T. and Tanaka, T. (1993) Discrete Particle Simulation of Two-Dimensional Fluidized Bed. Powder Technology, 77, 79-87. https://doi.org/10.1016/0032-5910(93)85010-7

[11] Kogane, A., Kuwagi, K. and Hirano, H. (2019) Dimensionless Parameter for Distinction between Homogeneous and Bubbling Fluidization Regimes. Advances and Applications in Fluid Mechanics, 23, 69-96. https://doi.org/10.17654/FM023010069

[12] Valverde, J.M., Castellanos, A., Mills, P. and Quintanilla, M.A.S. (2003) Effect of Particle Size and Interparticle Force on the Fluidization Behavior of Gas-Fluidized Beds. Physical Review E, 67, Article ID: 051305.

https://doi.org/10.1103/PhysRevE.67.051305

[13] Ye, M., van der Hoef, M.A. and Kuipers, J.A.M. (2005) The Effects of Particle and Gas Properties on the Fluidization of Geldart A Particles. Chemical Engineering Science, 60, 4567-4580. https://doi.org/10.1016/j.ces.2005.03.017

[14] Di Renzo, A. and Di Maio, F.P. (2007) Homogeneous and Bubbling Fluidization Regimes in DEM-CFD Simulations: Hydrodynamic Stability of Gas and Liquid Fluidized Beds. Chemical Engineering Science, 62, 116-130. https://doi.org/10.1016/j.ces.2006.08.009

[15] Thornton, C., Yang, F. and Seville, J. (2015) A DEM Investigation of Transitional Behavior in Gas-Fluidized Beds. Powder Technology, 270, 128-134. https://doi.org/10.1016/j.powtec.2014.10.017

[16] Foscolo, P.U., Di Felice, R., Gibilaro, L.G., Pistone, L. and Piccolo, V. (1990) Scaling Relationships for Fluidisation: The Generalised Particle Bed Model. Chemical En- 
gineering Science, 45, 1647-1651. https://doi.org/10.1016/0009-2509(90)80018-A

[17] Rietema, K. and Piepers, H.W. (1990) The Effect of Interparticle Forces on the Stability of Gas-Fluidized Beds-I. Experimental Evidence. Chemical Engineering Science, 45, 1627-1639. https://doi.org/10.1016/0009-2509(90)80015-7

[18] Jacob, K.V. and Weimer, A.W. (1987) High-Pressure Particulate Expansion and Minimum Bubbling of Fine Carbon Powders. AIChE Journal, 33, 1698-1706. https://doi.org/10.1002/aic.690331013

[19] Xie, H.Y. and Geldart, D. (1995) Fluidization of FCC Powders in the Bubble-Free Regime. Powder Technology, 82, 269-277.

https://doi.org/10.1016/0032-5910(94)02932-E 


\section{Nomenclature}

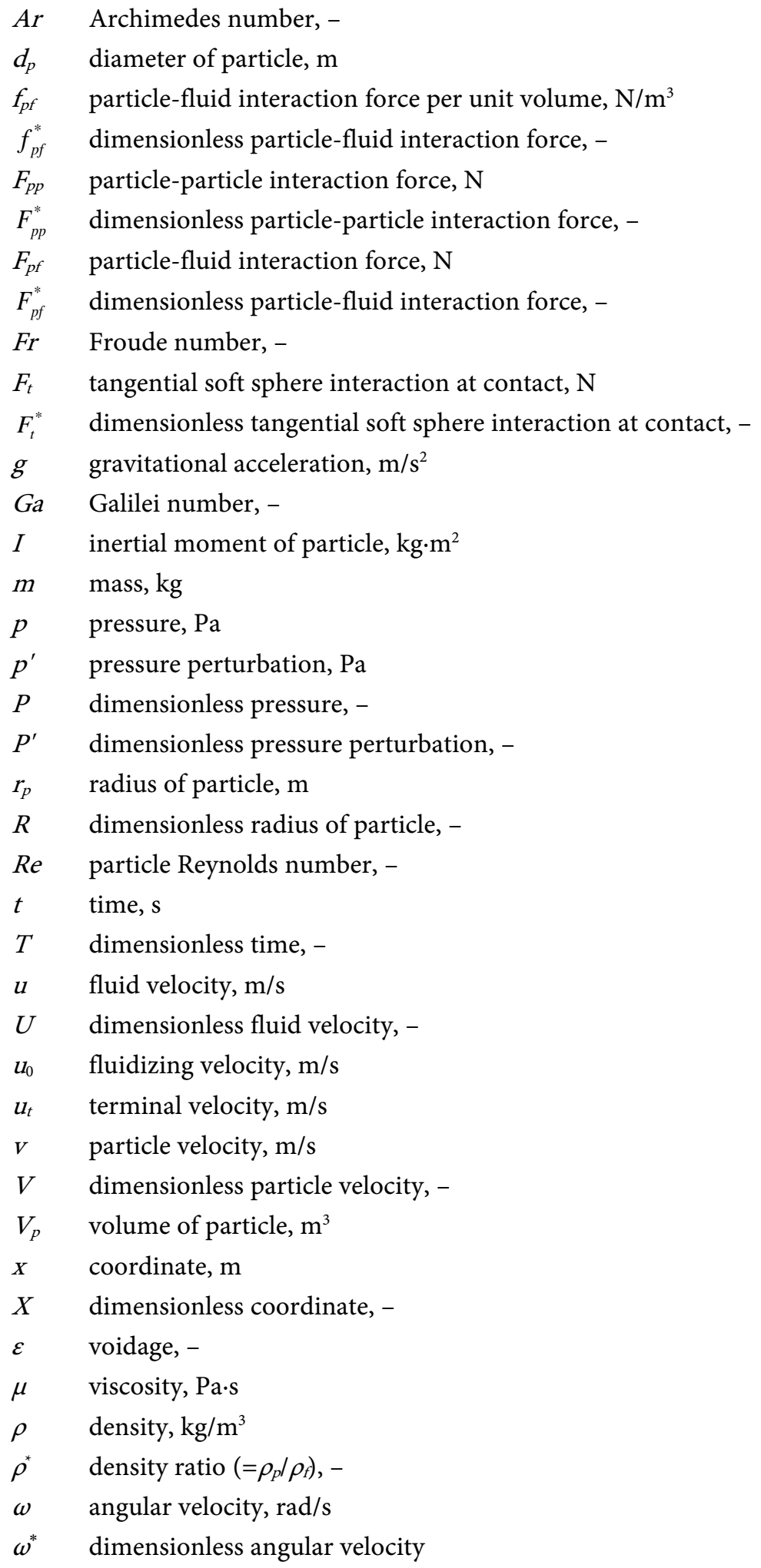

\section{Subscripts}

$f \quad$ fluid 


$$
\begin{array}{ll}
m f & \text { minimum fluidization } \\
m b & \text { minimum bubbling fluidization } \\
o & \text { arbitrary reference quality } \\
p & \text { particle }
\end{array}
$$

